18 | 2014

NOVECENTO... E DINTORNI

Da Torino a Parigi: Laura Malvano storica e critica d'arte

\title{
Michelangelo riciclato: Il Rinascimento nella cultura di massa
}

Michel-Ange recyclé: la Renaissance dans la culture de masse

Michelangelo Recycled: Renaissance in Contemporary Mass Culture

\section{Luciano Cheles}

\section{OpenEdition \\ Journals}

Edizione digitale

URL: http://journals.openedition.org/cei/1704

DOI: 10.4000/cei.1704

ISSN: 2260-779X

\section{Editore}

UGA Éditions/Université Grenoble Alpes

\section{Edizione cartacea}

Data di pubblicazione: 30 mars 2014

Paginazione: 117-129

ISBN: 978-2-84310-268-4

ISSN: $1770-9571$

Notizia bibliografica digitale

Luciano Cheles, «Michelangelo riciclato: II Rinascimento nella cultura di massa», Cahiers d'études italiennes [Online], 18 | 2014, online dal 30 septembre 2015, consultato il 26 mars 2021. URL: http:// journals.openedition.org/cei/1704 ; DOI: https://doi.org/10.4000/cei.1704 


\title{
MICHELANGELO RICICLATO: \\ IL RINASCIMENTO NELLA CULTURA DI MASSA
}

\author{
Luciano Cheles
}

L'arte non è più l'appannaggio esclusivo di gruppi ristretti di specialisti (studenti e studiosi di storia dell'arte, artisti e collezionisti), ma appassiona anche il grande pubblico. Questo interesse si è sviluppato soprattutto dal dopoguerra in poi: il turismo di massa, i musei sempre più accoglienti (tanto da diventare a volte vere e proprie strutture ricreative), le mostre spettacolari, la proliferazione di volumi d'arte riccamente illustrati, i documentari televisivi e i film che s'ispirano alla vita di artisti sono allo stesso tempo l'origine e il risultato di questo fenomeno culturale.

La democratizzazione dell'arte ha condotto ad una parziale perdita dell'aura che l'avvolgeva: con i «capolavori» del passato abbiamo ormai un rapporto di familiarità, che ci incoraggia a riciclarli con finalità diverse rispetto a quelle originarie, spesso in modo ludico e irriverente, tramite procedimenti quali la citazione, la parodia, l'allusione e il pastiche. Sono le opere del Rinascimento ad essere appropriate con particolare frequenza. Ciò avviene non solo per la loro originalità figurativa e qualità tecnica, ma anche per la loro bellezza e sensualità, che le rendono particolarmente appetibili ai media contemporanei. Queste opere, che ci vengono riproposte sia esplicitamente che allusivamente, sono di un'efficacia potenziale considerevole: essendo sedimentate nella nostra memoria visiva collettiva, siamo naturalmente predisposti ad apprezzarle ${ }^{\mathrm{I}}$.

I. Sul riuso delle opere d'arte nella comunicazione di massa vi è un'ampia bibliografia. Si veda per es.: D. Schneider, Art-Pub. La pub détourne l'art, Genève, Tricorne (éditions Dru), I999; B. Hoffman, The Fine Art of Advertising, New York, Stewart, Tabori and Chang, 2000; L. Flutsch e C. M. Pruvot (prefaz.), in Recycled Past. Antiquity in Contemporary Marketing, Lausanne, Musée national suisse, I997; B. e F. Schnitzler, Archéopub. La survie de l'antiquité dans les objets publicitaires, Strasbourg, Musée de Strasbourg, 2006; M. Centanni (a cura di), Classico manifesto. Temi della tradizione classica nella pubblicità italiana, Roma, Edizioni Fondazione Valore Italia, 2008; D. SAlmon, La Vénus de Milo: un mythe, Paris, Gallimard, 2000; A. Chastel, L'illustre incomprise. Mona Lisa, Paris, Gallimard, 1988; G. Roque, Ceci n’est 
Michelangelo è tra gli artisti più sfruttati dalla cultura figurativa di massa; da qui la scelta di consacrare una parte importante del presente saggio a immagini che fanno riferimento alle sue opere.

La pubblicità degli stilisti e degli empori specializzati in capi d'abbigliamento si è spesso servita dell'iconografia rinascimentale. D'altronde i rapporti tra moda e arte sono stretti, non solo perché ambedue appartengono alla sfera visiva, ma perché lo stilista, proprio come l'artista, crea un oggetto la cui principale funzione è quella di piacere, anziché essere utile ${ }^{2}$.

Il David della Galleria dell'Accademia ricorre con particolare frequenza. Compare da qualche anno, appositamente «vestito», nei manifesti e negli opuscoli pubblicitari dei grandi magazzini francesi BHV (Bazar de l'Hôtel de Ville) (fig. I), per sottolineare la qualità degli indumenti, nonché per suggerire umoristicamente che trasformeranno l'acquirente in un uomo aitante come l'eroe michelangiolesco. Con simili finalità la Levis si è servita della scultura in questione, nonché dell'affresco della Creazione di Adamo, per commercializzare i suoi jeans ${ }^{3}$. L'immagine di Dio, che dopo aver creato il primo uomo gli intima di coprire le proprie nudità con l'indumento della ditta è, perlomeno storicamente, tutt'altro che inappropriata, poiché, com'è noto, poco dopo il Concilio di Trento, la Cappella Sistina fu censurata: le nudità delle figure furono coperte con panneggi e foglie di fico da Daniele da Volterra, che si guadagnò così il soprannome di «Braghettone».

Il David di Michelangelo si ritrova nella pubblicità di altri prodotti e servizi che riguardano l'estetica corporale. Il suo viso è stato riprodotto, per esempio, nel 2007, sulla stampa di Boston per pubblicizzare una clinica di cosmesi dentale (scelta un poco azzardata visto che le sue labbra sono serrate), mentre il corpo imberbe dell'eroe è stato raffigurato per intero su alcune riviste londinesi (c. 2003) per pubblicizzare un centro di epilazione che - pare promettere - renderà il corpo del cliente liscio come il marmo della statua.

pas un Magritte. Essai sur Magritte et la publicité, Paris, Flammarion, 1983. Questa letteratura tratta quasi esclusivamente di immagini artistiche riprese in modo palese. Il presente saggio si soffermerà anche sul fenomeno della rivisitazione velata dei capolavori.

2. Sui rapporti tra moda e arte, si veda: F. Zeri, Radici molto antiche, e M. Marchetti, Art couture, ambedue in Maison Valentino (a cura di), Valentino. Trent'anni di magia. Le opere, Milano, Leonardo, I990, rispett. pp. I7 e 34-35; O. Calabrese, Lo stile dei sensi, in G. Versace e O. Calabrese, Vanitas, Milano, Leonardo, I99I, pp. 7-I7. Per l'impatto di movimenti artistici sulla moda, si veda per esempio: E. CrispolTI, Il Futurismo e la moda, Venezia, Marsilio, I988; V. Guillaume (a cura di), Europe 1910-I939. Quand l'art habillait le vêtement, Paris, Paris Musées, I997; C. Brändstatter, Klimt et la mode, Paris, Éd. Assouline, I998; F. Boudot, Mode et surréalisme, Paris, Éd. Assouline, 2002.

3. Google Immagini > Levis Michelangelo. 


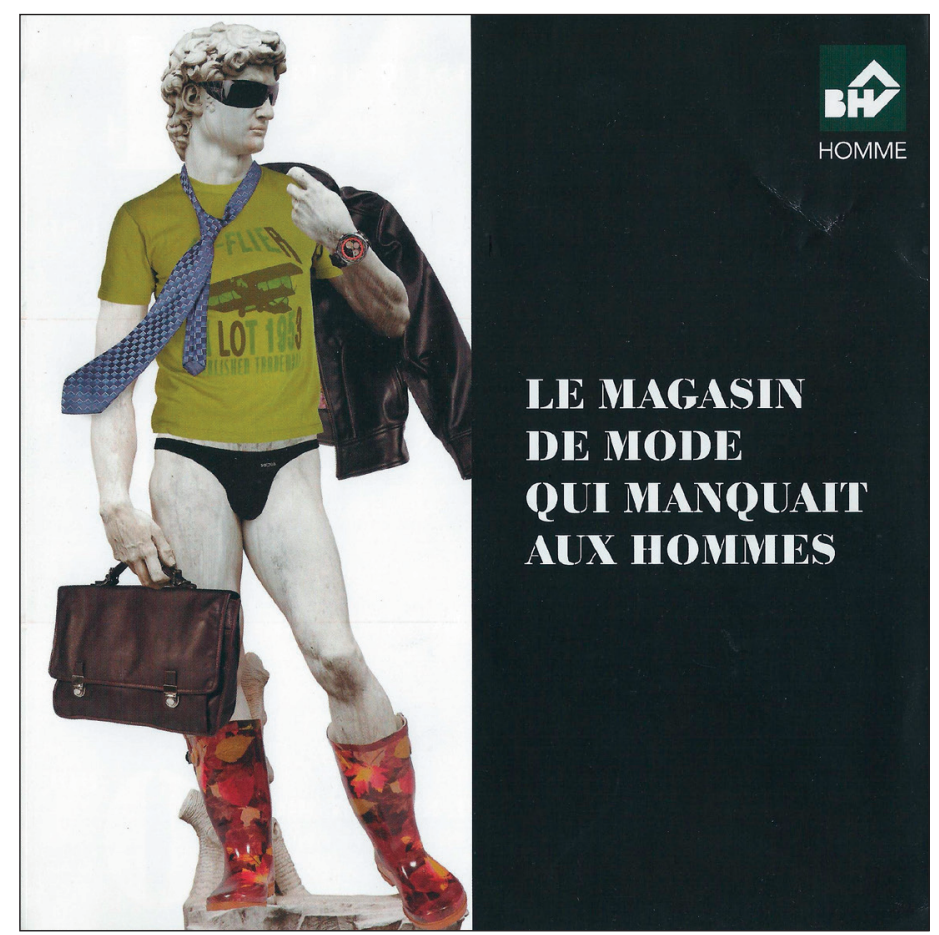

Fig. I. - Manifesto e opuscolo pubblicitario dei grandi magazzini BHV (Bazar de l'Hôtel de Ville), Parigi, 20IO-20I2.

Spesso gli stilisti si servono anche dell'iconografia religiosa, e più precisamente di quella relativa alla Passione, per pubblicizzare i loro prodotti. Un annuncio della Biblos (1990), ditta specializzata in indumenti intimi, raffigura un uomo con la testa china e le braccia incrociate sopra il capo come se fossero legate davanti. Questi particolari sono talmente insoliti che è legittimo ritenere che possano essere significativi. L'immagine si ispira liberamente all'iconografia dell'Ecce homo, che raffigura Cristo coronato di spine, con il torso nudo coperto da un mantello e un giunco inserito a mo' di ironico scettro tra le mani legate davanti ${ }^{4}$. Segue più fedelmente quest'iconografia una foto pubblicitaria per gli abiti Istante (1995). Essa raffigura infatti un giovane con i baffi, il pizzo, l'espressione malinconica, il torso nudo parzialmente coperto da una giacca quasi fosse un manto, e le mani che si uniscono davanti per tenere la stecca di biliardo. Quest'ultima, che sostrituisce la canna, è stata qui usata con evidenti sottintesi sessuali.

4. Si veda L. RÉAu, Iconographie de l'art chrétien, tomo II, vol. II. Iconographie de la Bible. Nouveau Testament, Paris, P.U.F., 1957, pp. 459-46I. 
Anche il tema della Pietàs è stato più volte fatto proprio dalla pubblicità di moda. Un manifesto della List (1995), ad esempio, raffigura una giovane donna vestita che regge teneramente la testa di un ragazzo disteso a torso nudo. Lo stesso soggetto è rinvenibile in una pubblicità per il profumo maschile Gucci Envy (1998) ${ }^{6}$ e in un manifesto della Kookai $(200 \mathrm{I})^{7}$. Se queste immagini alludono chiaramente alla Pietà vaticana, l'annuncio pubblicitario della Calvin Klein raffigurante una donna che cavalca suggestivamente un uomo $(2002)^{8}$ s'ispira invece a un'altra celebre Pietà michelangiolesca, quella di Palestrina (Galleria dell'Accademia, Firenze) raffigurante la Vergine che sostiene da dietro il corpo muscoloso nudo di Cristo. Queste immagini sfruttano la stretta connessione tra sesso e morte, ossia tra la temporanea perdita di coscienza nel momento culminante dell'amplesso (la petite mort, come la chiamano i francesi), e quell'assenza permanente che è la morte.

È di particolare interesse un'immagine per i jeans di Valentino che raffigura frontalmente un uomo disteso a torso nudo (I990) (fig. 2). Essa si ispira palesemente al Cristo scorto del Mantegna (Brera). Il riferimento a una delle opere più intensamente religiose del Rinascimento può sembrare inappropriato e addirittura blasfemo, visto che lo scopo della fotografia è quello di attirare il nostro sguardo sulla regione pubica della figura. Se l'intento provocatorio è indubbio (come lo è nelle precedenti immagini mutuate dalle iconografie della Pietà), va rilevato che probabilmente anche il dipinto del Mantegna vuole invitarci a focalizzare il nostro sguardo sulle parti intime di Cristo. Leo Steinberg ha difatti convincentemente argomentato, in un volume dal titolo La sessualità di Cristo nell'arte rinascimentale e il suo oblio nell'epoca moderna, che le rappresentazioni rinascimentali di Gesù spesso sottolineano, tramite accorgimenti diversi, la sua virilità - un'enfasi che serviva a simbolizzare la natura terrena del figlio di Dio, e cioè a illustrare il mistero del Verbo fatto Carne?

5. Ivi, tomo II, vol. II, pp. 5I9-52I; e J. Turner (a cura di), The Dictionary of Art, London, Macmillan Publishers, I996, vol. 24, s. v. «Pietà», pp. 775-777.

6. Google Immagini > Gucci Envy for men. Qui la figura maschile che allude al Cristo della Pietà è raffigurata da sola.

7. Google Immagini > Kookai Pietà.

8. Google Immagini > Calvin Klein Woman riding man in briefs.

9. Il volume (Milano, 1986), è la traduzione della prima edizione di The Sexuality of Christ in Renaissance Art and in Modern Oblivion, New York, Pantheon Books, 1983. Di questa opera è apparsa a Chicago nel 1996 un'edizione riveduta e ampliata. Su un simile tema si veda anche A. Leupin, Phallomanies. La chair et le sacré, Paris, Éd. du Regard, 2000. 


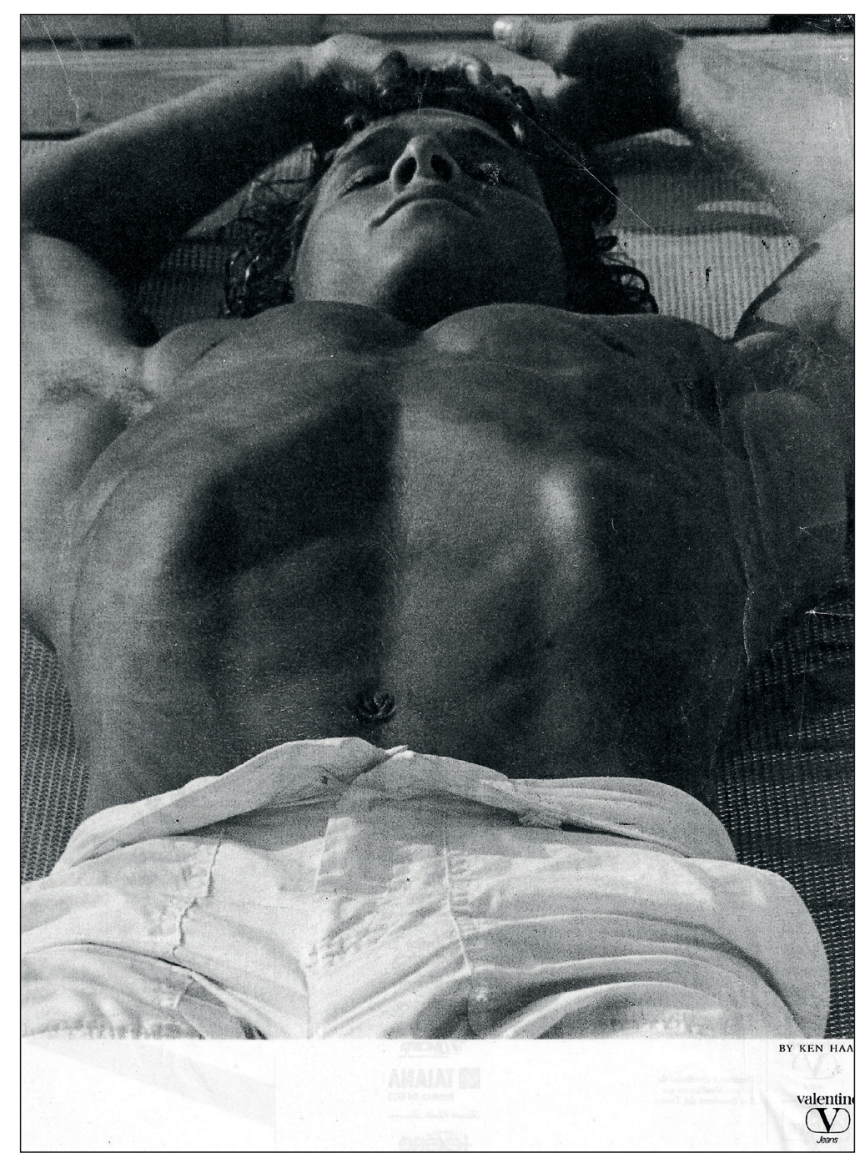

Fig. 2. - Annucio pubblicitario, Valentino, I990.

Fotografia di Ken Haak.

Un annuncio pubblicitario per il profumo di toeletta Drakkar Noir della Guy Laroche (1995), e un altro per un'Acqua di Colonia Dolce e Gabbana (2002), che raffigurano ambedue un uomo a torso nudo apparentemente noncurante delle attenzioni della ragazza che gli sta alle spalle, sembrano anch'esse ispirarsi al tema della Pietà. Più precisamente, esse evocano la tipologia che raffigura il Cristo morto ma in posizione eretta, con a fianco la Vergine che lo piange e lo bacia, di cui uno degli esempi più noti è la Pietà di Giovanni Bellini che si conserva a Brera ${ }^{\mathrm{I}}$.

Io. Per questa iconografia, si veda E. Panofsky, Imago Pietatis. Contribution à l'histoire des types du "Christ de Pitié" / "Homme de Douleurs" et de la "Maria Mediatrix", in ID., Peinture et dévotion en Europe du Nord à la fin du Moyen Âge, Paris, Flammarion, 1997, pp. 13-28, I25-136, figg. I-22. 
Gesù non è l'unico martire a cui alludano le immagini degli stilisti. Alcune rappresentazioni di ragazzi più o meno svestiti che volgono la testa verso l'alto si rifanno all'iconografia di San Sebastiano ${ }^{\text {II }}$. È particolarmente interessante quella prodotta nel 1988 per la camiceria Fenicia (fig. 3). La figura maschile sembra ispirarsi al San Sebastiano mantegnesco della $\mathrm{Ca}$ d'Oro di Venezia, come suggeriscono il contrapposto e il panneggio «inamidato». Per evitare che la fonte traspaia in modo esplicito, la postura del santo è stata riprodotta al contrario. Tuttavia c'è un particolare del portamento della figura che non appartiene all'iconografia del santo: la mano destra che cela il pube con il lembo della camicia. Si tratta di un gesto troppo aggraziato per essere virile. Il motivo appartiene alla Venus pudica, un genere iconografico che trae origine dall'antichità greco-romana e fu frequentemente imitato nel Rinascimento (basti ricordare la Nascita di Venere del Botticelli e la Venere di Urbino del Tiziano, ambedue negli

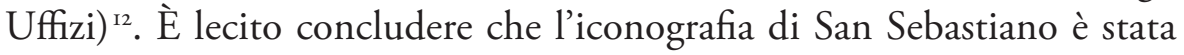
qui interpretata ironicamente, con gusto post-moderno: l'atletico santo, diventato nel Rinascimento, come vedremo, l'incarnazione della bellezza maschile, è stato femminilizzato attraverso l'allusione a Venere. L'immagine vuole esprimere l'idea scherzosa che le camicie Fenicia rendono i ragazzi che le indossano avvenenti come... Venere! L'idea è meno peregrina di quanto possa sembrare: nel 1987 una pubblicità del maglificio Fiume raffigurava, sullo sfondo della Nascita di Venere del Botticelli, un giovane atteggiato come la dea dell'amore. La figura retorica utilizzata in queste immagini è l'ossimoro, una delle più apprezzate nella cultura classica e rinascimentale ${ }^{13}$.

II. Sull'iconografia di San Sebastiano, la letteratura è ampia. Si veda soprattutto: F. Le Targat, Saint Sébastien dans l'histoire de l'art depuis le XV siècle, Paris, J. Damase, I979; J. Darriulat, Sébastien le Renaissant, Paris, Lagune, I999; K. Ressouni-Demigneux, Saint Sébastien, Paris, Éd. du Regard, 2000.

I2. Su questa tipologia classica e il suo impatto sull'arte rinascimentale (e quella dei periodi successivi), si veda: P. B. Bober \& R. O. Rubinstein, Renaissance Artists and Antique Sculpture. A Handbook of Sources, London, Oxford University Press, 1986, pp. 59-6I; N. Salomon, "The Venus Pudica". Uncovering Art History's "Hidden Agendas" and Pernicious Pedigrees, in G. Pollock (a cura di), Generations and Geographies in the Visual Arts. Feminist Readings, London, Routledge, 1996, pp. 69-88.

13. R. L. Colie, Paradoxia Epidemica. The Renaissance Tradition of Paradox, Princeton, Princeton University Press, 1966; E. WInd, Misteri pagani del Rinascimento, Milano, Adelphi, I985, pp. I06-I40. 


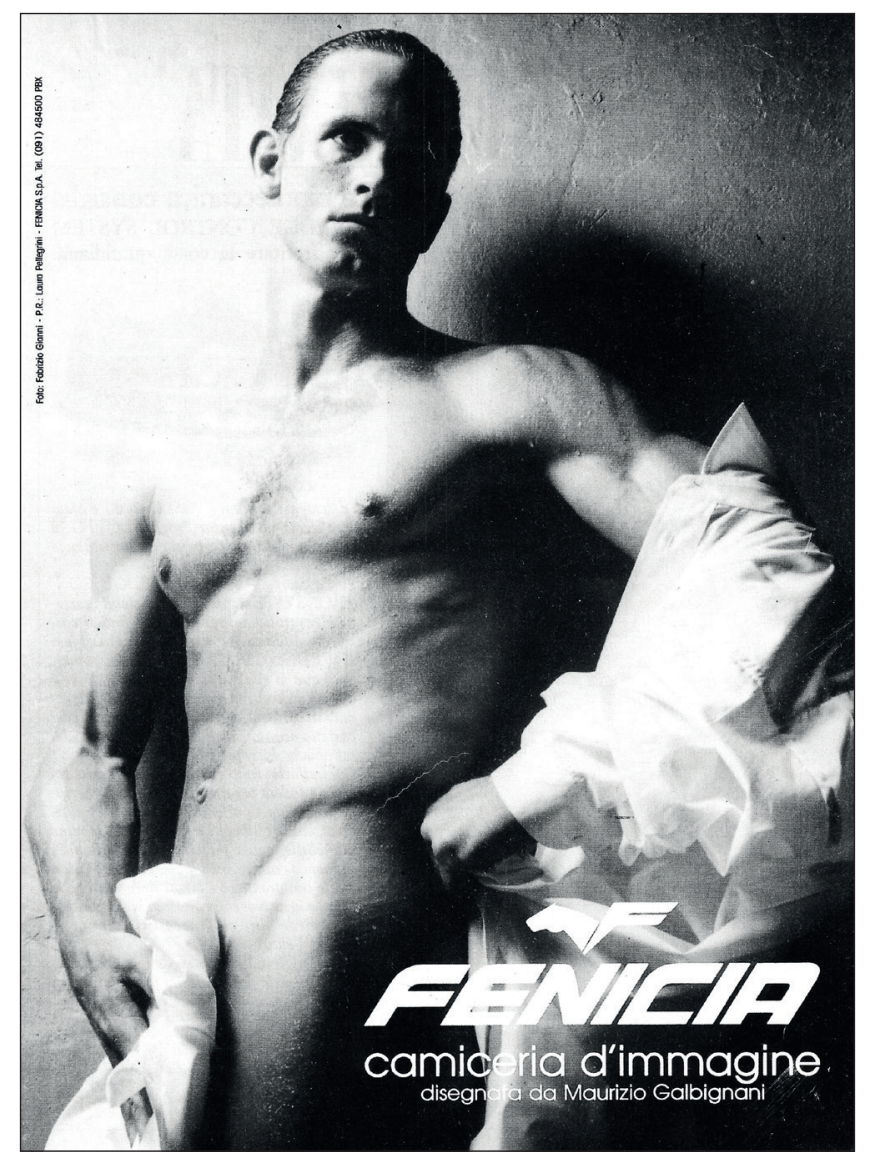

Fig. 3. - Annucio pubblicitario, Fenicia, 1988. Fotografia di Fabrizio Gianni.

L'appropriazione di tipologie cristiane, e più precisamente del tema del martirio, da parte della pubblicità degli stilisti può sorprendere e merita quindi una spiegazione. E noto che nelle rappresentazioni artistiche i martiri hanno solitamente un fisico bello, slanciato e vigoroso, sebbene nel mondo reale il corpo di un uomo o di una donna che ha subito la violenza fisica più estrema possa solo ispirare orrore. Ciò è dovuto al fatto che nella tradizione classica e poi cristiana il corpo atletico e avvenente simbolizzava il trionfo della virtù. La possanza fisica e la bellezza erano i segni del sostegno divino. Poiché originariamente il termine virtus significava sia «mascolinità» che "coraggio» (vir e virtus hanno la stessa etimologia), l'immagine dell'atleta aitante finì con l'essere utilizzata per tradurre il concetto di virtù morali e cristiane. L'esaltazione della forza fisica offriva 
dei vantaggi: permetteva alla Chiesa di sottolineare non la sofferenza del martire, ma la sua straordinaria capacità di resistenza nella contesa tra torturatore e vittima - una contesa in cui alla fine è il martire a trionfare ${ }^{\mathrm{I}}$.

Non diversamente dagli artisti del Rinascimento, che si dilettavano molto a rappresentare il corpo bello straziato dalle torture perché questo soggetto permetteva loro di sfoggiare la loro capacità di rendere realisticamente l'anatomia umana in pose drammatiche, i fotografi degli stilisti si ispirano all'iconografia del martirio con finalità a loro proprie, e cioè per sfruttarne il contenuto erotico esplicito o sottinteso. Dei numerosi santi-martiri è soprattutto Sebastiano ad essere utilizzato dalla pubblicità di capi d'abbigliamento perché è il santo più aitante, il più "palestrato». Il Rinascimento ne fece il simbolo della bellezza maschile. In quanto milite (secondo la tradizione, era un capitano della guardia pretoriana dell'Imperatore Diocleziano), il giovane Sebastiano si prestava a essere raffigurato con corporatura atletica. Inoltre, la natura stessa del suo martirio lo predisponeva a essere rappresentato come oggetto sessuale passivo. Legato e seminudo, è al centro dell'attenzione dei suoi sicari, le cui frecce puntate sul suo corpo fungono da metafora del desiderio ${ }^{15}$. Si aggiunga che in generale il corpo atletico, se accompagnato da un'espressione malinconica e sofferente, risulta fortemente erotico. Attrae perché offre il piacere visivo di un'anatomia possente senza tuttavia la minaccia di violenza fisica che normalmente si associa ad essa. Infine l'immagine del macho-triste, del fusto-fragile, in altre parole dell'aitante giovane che non si vergogna di mostrare la sua vulnerabilità, fa appello all'istinto materno ${ }^{16}$. Che i pubblicitari rovistino nel repertorio iconografico del martirio per creare immagini positive e attraenti di figure depresse, umiliate e sofferenti poco sorprende. Dopotutto dobbiamo proprio al Cristianesimo l'invenzione dell' «iconografia masochista», e cioè di un immaginario che presenta la punizione e la sofferenza come piacere (in quanto facilita la conquista della beatitudine eterna).

La celebrazione dell'uomo in pena, vulnerabile e passivo pare accordarsi bene con l'etica attuale della political correctness. Questo soggetto ci viene presentato come l' «uomo nuovo» che le femministe vagheggiano

I4. Si veda l'importante studio di C. EIsler, The Athlete of Virtue. The Iconography of Asceticism, in M. Meiss (a cura di), De Artibus Opuscula. XL. Essays in Honor of Erwin Panofsky, New York, Buehler Buchdruck, 196I, vol. I, pp. 82-97 (testo), vol. II, pp. 23-26 (illustr.).

I5. J. Darriulat, Sébastien le Renaissant, cit., cap. IX («Le séducteur»), spec. p. 20 .

I6. Faccio mie le interessanti osservazioni di A. Foster, Heroes, Fools, and Martyrs, "Ten-8», n 28, I988, pp. 54-63. Di Foster va citato anche l'importante volume Behold the Man. The Male Nude in Photography, Edinburgh, Sills Gallery, 1988. Sulla carica erotica che la figura del «bel malinconico» può suscitare, si veda R. Dyer, Coming out as going in, in ID., The Matter of Images. Essays on Representations, London, Routledge, I993, pp. 73-92. 
da decenni - un uomo che ha scoperto il suo lato femminile. Le immagini che raffigurano muscolosi ragazzi in atteggiamenti femminili devono interpretarsi come un'allusione a queste recenti tendenze. Le raffigurazioni di una mascolinità languida, ansiosa e fragile si vogliono quindi progressiste. Si presentano come un salutare antidoto a quelle convenzionali dell'uomo forte, eretto, sicuro di sé, in altre parole, dell'uomo «fallico» che ha dominato la pubblicità fino agli anni Ottanta.

Queste immagini mutuate dall'arte rinascimentale compaiono perlopiù sulla stampa di qualità. Aspirano a lusingare il pubblico di cultura medioalta a cui si rivolgono invitandolo a cogliere e apprezzare, magari subliminalmente, le allusioni artistiche che contengono. Il pubblico in questione non è esclusivamente maschile: è noto che profumi e abbigliamento intimo sono spesso acquistati dalle donne per i loro uomini. Le donne «moderne e liberate» sono tenute ad apprezzare questo tipo di immagini per le ragioni sovraesposte. Quanto agli uomini, è lecito ritenere che questo genere di pubblicità che pare umiliare la loro virilità non dispiaccia loro. Si tratta, dopotutto, di un gioco intelligente basato sull'auto-ironia, un gioco che presuppone da parte di chi è sollecitato a parteciparvi la certezza più assoluta sulla propria mascolinità, e che quindi non può che lusingare. Inoltre, come si è visto, il vittimismo rende: l'immagine del macho-molle, del forzuto-depresso risulta altamente appetibile alle donne. Vale infine la pena di ripetere che nel tema della vittima innocente è implicito quello del trionfo. È dunque legittimo nutrire qualche dubbio riguardo alla presunta modernità e alla political correctness di immagini che, ispirandosi all'iconografia del martirio, rappresentano il maschio che si crogiola nella sofferenza per essere venerato da una donna come un dio.

Nella pubblicità commerciale i capolavori del Rinascimento sono talvolta citati esplicitamente non solo per celebrare la buona qualità e il bel design dei prodotti, bensì anche per suggerire l'idea di italianità. Per esempio, un'immagine pubblicitaria per Vinitaly, la fiera dei produttori dei vini italiani, riproduce il motivo delle mani della Creazione d'Adamo (1996); un'altra per Asti Spumante si serve del Tondo Pitti di Michelangelo (Bargello, Firenze, I99I), sebbene il vino in questione sia piemontese anziché toscano; un'altra ancora annuncia una mostra di artigianato e specialità gastronomiche italiane alla stazione di Bercy (Parigi) servendosi del David (2002); quella degli orologi Veglia cita invece il Mosé di Michelangelo (Basilica di San Pietro in Vincoli, Roma, 1996), forse per sottintendere che gli svizzeri non hanno il monopolio del design orologistico, e che il paese che ha dato i natali a Michelangelo è ben capace di eccellere anche nel campo in questione. 


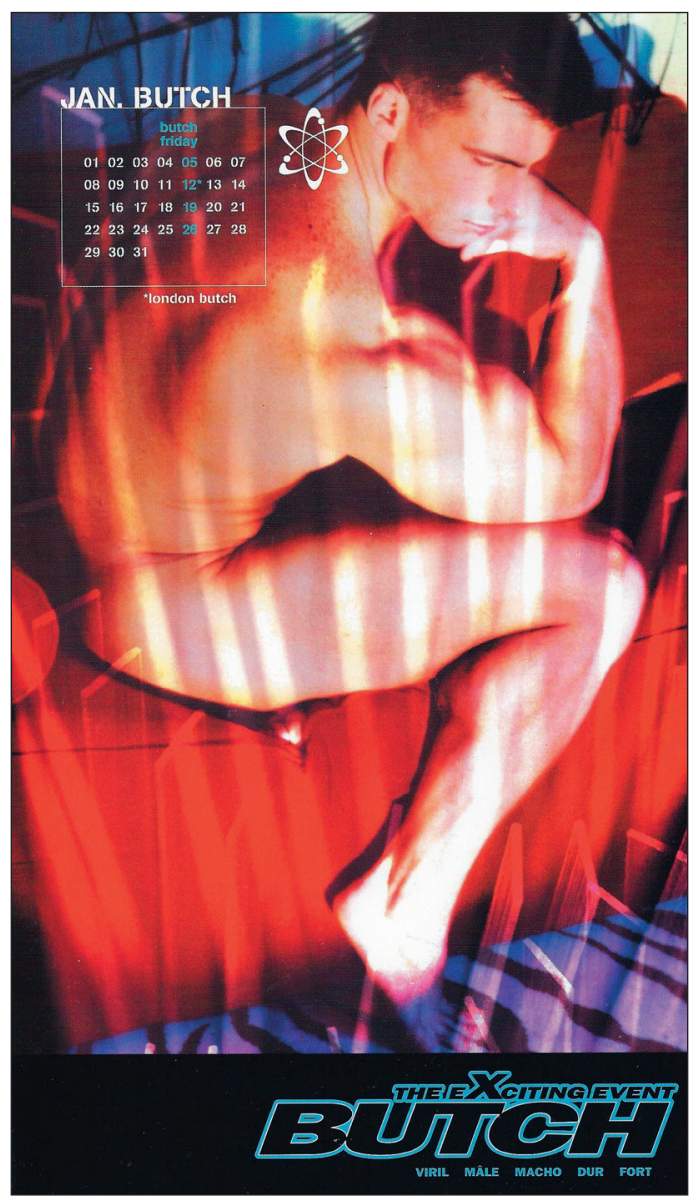

Fig. 4. - Volantino pubblicitario per una discoteca, Parigi, 2007.

I nudi michelangioleschi sono stati appropriati a dei fini libertari nonché libertini. Un network americano ha utilizzato il David per promuovere una serie di programmi culturali che trattano tematiche sessuali (c. 2005): qui l'eroe è stato raffigurato con uno slip abbassato per affermare che l'arte non si censura ${ }^{17}$. Un David dall'attributo maschile sovrasviluppato compare nei fumetti erotici del celebre grafico Touko Laaksonen, alias Tom of Finland (anni 80) ${ }^{18}$. Infine, sui volantini che pubblicizzano i locali notturni gay parigini sono talvolta raffigurate figure nude in pose

I7. B. Hoffman, The Fine Art, cit., p. 44.

I8. Google Immagini > Tom of Finland Michelangelo's David. Su questo illustratore, cfr. V. Morlock, Tom of Finland, Los Angeles, Tom of Finland Foundation, 1997. 
languide che si ispirano alle sculture delle Cappelle Medicee di Firenze (fig. 4). Un'altra celebre scultura michelangiolesca, lo Schiavo morente del Louvre, decora con gusto post-moderno i balconi degli ultimi due piani di una costruzione dell' ${ }^{\circ}{ }^{\circ}$ arrondissement parigino, realizzata nel I99I dall'architetto Manolo Núńez Yanowsky ${ }^{19}$. La reiterazione del motivo dello schiavo, e cioè di un prigioniero, serve a ricordarci con umorismo che l'edificio in questione ospita il commissariato di polizia.

Alcune opere di Michelangelo sono state inoltre utilizzate a fini politici e sociali. Si rifa al David una scultura di Arno Breker, lo scultore ufficiale del III Reich, intitolata Vigilanza (1939) (fig. 5) ${ }^{20}$. Michelangelo non poteva non piacere a Breker: la sua «terribilità» e i suoi nudi sovrumani erano difatti in sintonia con il culto nazista del gigantismo e della forza virile.

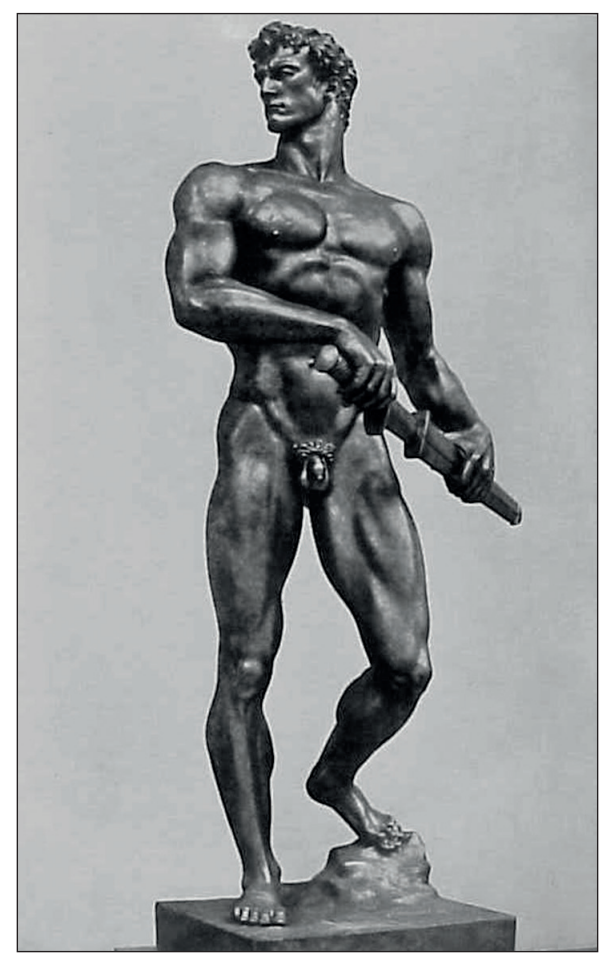

Fig. 5. - Arno Breker, Vigilanza, 1939, Museo Arno Breker, Schloss Nörvenich (presso Colonia).

19. Google Immagini > Manolo Núñez Yanowsky esclaves mourants.

20. Su Breker, Cfr. J. M. Infiesta, Arno Breker: Der Michelangelo des XX. Jahrhunderts, Tübingen, o. A., o. A., I982; J. Trimborn, Arno Breker. Der Kunstler und die Macht. Die Biographie, Berlin, Aufbau Verlag, 2OII. 
La Pietà compare nella propaganda soprattutto per illustrare i temi della sofferenza e della solidarietà ${ }^{21}$. Un manifesto diffuso dalla Democrazia Cristana nel 1957 per commemorare la Liberazione raffigura una madre che sostiene il figlio morto nella stessa posa della Vergine con Gesù (fig. 6). Esso non si limita però a celebrare il martirio dei partigiani: il testo invita infatti il pubblico a non dimenticare che è grazie ad essi che la democrazia è stata ristabilita e lo sollecita ad opporsi al comunismo. Lo scopo ultimo di questa Pietà moderna è quello di negare alla sinistra il suo ruolo chiave nella Resistenza, dando addirittura l'impressione che i comunisti, tradizionalmente rappresentati come atei e anti-clericali, siano i nemici contro cui i partigiani si sono battuti. Un altro manifesto, diffuso dal Movimento Sociale Italiano nel 1970, si rifa alla Pietà di Michelangelo per invitare a firmare una petizione a favore della pena di morte per gli spacciatori di droga. La vittima di un'overdose vi è difatti rappresentata nella posa di Gesù. L'allusione alla scultura di Michelangelo serve a colpire lo spettatore sia emotivamente che "artisticamente" per impedirgli di ragionare sulla gravità della proposta che gli viene fatta. Inoltre l'identificazione del giovane con il Cristo martoriato suggerisce che la gravità del crimine giustifica l'introduzione della pena capitale ${ }^{22}$.

La Pietà è frequentemente utilizzata anche dalla pubblicistica di protesta: la troviamo riprodotta su un manifesto del 2003 , realizzato dall'International Solidarity Movement per esprimere indignazione per il massacro dei palestinesi perpetrato dall'esercito israeliano ${ }^{23}$. Infine è interessante notare che il motivo focale della Creazione di Adamo è stato riprodotto su un manifesto del movimento di protesta cinese New Democracy, realizzato dagli studenti dell'Accademia di Belle Arti di Beijing nel 1989. L'immagine è accompagnata dalla scritta «Il popolo unito. Ai giovani che il 13 maggio I989 si sono impegnati in uno sciopero della fame per la democrazia» ${ }^{24}$. Le due mani che si sfiorano vogliono probabilmente rappresentare la volontà dei sostenitori della democrazia, che il regime ha disperso e represso, di

2I. Su questa tematica, cfr. L. Cheles, L'uso delle immagini rinascimentali nell'iconografia del manifesto politico, in R. VArese (a cura di), Letture di storia dell'arte, Ancona - Bologna, Il Lavoro Editoriale, I988, pp. 43-48, 56-58 (i cui esempi si possono aggiungere a quelli citati nel presente saggio). Anche l'arte impegnata si è avvalsa dell'iconografia della Passione. Cfr. S. Sвоrgi (a cura di), Arte della libertà. Antifascismo, guerra e liberazione in Europa, 1925-1945, Milano, Mazzotta, 1995; e Corps crucifiés. Picasso, Bacon, Dix, de Kooning, Guttuso, Sutherland, Saura, Paris, Réunion des musées nationaux, Montréal, Musée des beaux-arts, 1992.

22. L. Cheles, L'immagine riciclata: camuffamenti, citazioni e plagi nella propaganda figurativa italiana del secondo dopoguerra, in M. Ridolfi (a cura di), Propaganda e comunicazione politica. Storia e trasformazioni nell'età contemporanea, Milano, Bruno Mondadori, 2004, pp. 260-27I.

23. M. Glaser e M. Ilic, The Design of Dissent, Gloucester, Rockport Publishers, 2006, p. I2.

24. L. McQuiston, Graphic Agitation. Social and Political Graphics since the Sixties, London, Phaidon, I993, pp. 82-83, fig. 3 . 
restare in contatto. Lo stesso motivo, ma con una delle due mani ridotta a uno scheletro, è stato utilizzato in un manifesto americano realizzato per mettere in guardia i tossicodipendenti contro il pericolo della condivisione di siringhe e aghi: qui, tuttavia, esso non ha il connotato positivo della prossimità, bensì l'idea sinistra del contagio ${ }^{25}$.

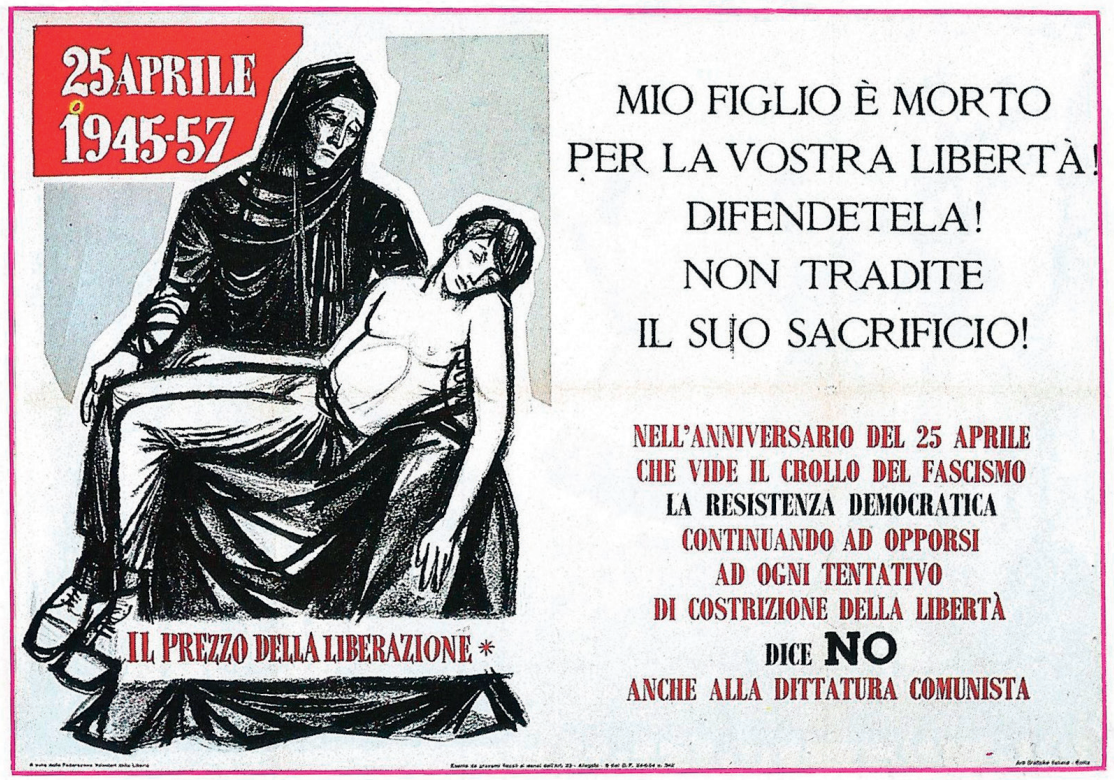

Fig. 6. - Manifesto della Democrazia Cristiana, 1957.

Impadronendosi delle opere d'arte più note, la cultura di massa inevitabilmente ne travisa il senso per soddisfare esigenze proprie. Queste alterazioni non devono tuttavia essere considerate come delle profanazioni, poiché il sincretismo High Brow e Low Brow produce spesso risultati estetici interessanti. Le citazioni, le parodie, le allusioni e le altre manipolazioni arricchiscono la cultura visiva di massa, senza nulla togliere alle opere originarie. 